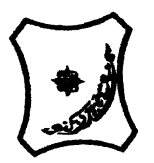

Bayero Journal of Pure and Applied Sciences, 11(2): 186 - 194

Received: October, 2018

Accepted: December, 2018

ISSN $2006-6996$

\title{
INFINITE INTEGRAL CONDITIONS FOR OSCILLATION OF FIRST ORDER LINEAR DELAY DIFFERENTIAL EQUATIONS WITH POSITIVE AND NEGATIVE COEFFICIENTS
}

*Tahir, A. and Haruna, Y.

Department of Mathematics, Modibbo Adama University of Technology Yola, Adamawa State, Nigeria Department of Mathematical Sciences Abubakar Tafawa Balewa University Bauchi, Bauchi State, Nigeria *Correspondence author: atahir@mautech.edu.ng

\section{ABSTRACT}

This study obtains a new set of integral conditions for oscillation of nonautonomous delay differential equations with positive and negative coefficients. It is however, discovered that every solution of the equation oscillates infinitely.

Keywords: Integral Condition, Oscillation, Positive and Negative Coefficients

\section{INTRODUCTION}

Delay differential equations are similar to ordinary differential equations but their evolution involves past values of the state variable. The solution of delay differential equations therefore, requires knowledge of not only the current state, but also of a certain time

$$
x^{\prime}(t)+p(t) x(t-\tau)=0, t \geq 0 .
$$

The purpose of this work is to consider a more generalized form of equation (1.1) and give a detailed description of the oscillatory solution of the generalized equation for infinite conditions.

The problem of establishing integral conditions for oscillation of delay differential equations has been

$$
x^{\prime}(t)+p(t) x(t-1)=0
$$

where $p(t)$ to be a continuous function on the interval $[0, \infty)$. He therefore, established some connections between (1.2) and more general delay equations and used those facts to prove some well - known oscillation results. Philos and Sficas (1998) set a new oscillation criterion for the delay differential equation

$$
x^{\prime}(t)+p(t) x(t-\tau(t))=0
$$

where $p$ and $\tau$ are nonnegative continuous real - valued functions on the interval $[0, \infty)$. Philos and Sficas (1998) also considered the function $T$ and defined it by

$$
T(t)=t-\tau(t) t \geq 0 \text { ， }
$$

which is increasing, such that

$\lim _{t \rightarrow \infty} T(t)=\infty$.

That criterion according to them concerned the case where

$\liminf _{t \rightarrow \infty} \int_{T(t)}^{t} p(s) d s \leq \frac{1}{e}$.

Stavroulakis (2004) considered first order linear delay differential equation

$$
x^{\prime}(t)+p(t) x(\tau(t))=0, \quad t \geq t_{0}
$$

and the second order linear delay equation

$$
x^{\prime \prime}(t)+p(t) x(\tau(t))=0, \quad t \geq t_{0}
$$

where $p$ and $\tau$ are continuous functions on $\left[t_{0}, \infty\right), p(t)>0, \tau(t)$ is non-decreasing, $\tau(t) \leq t$ for $t \geq t_{0}$ and $\lim _{t \rightarrow \infty} \tau(t)=\infty$. He therefore, presented several criteria for the first order equation, for instance 


\section{BAJOPAS Volume 11 Number 2 December, 2018}

when

$0<\liminf _{t \rightarrow \infty} \int_{\tau(t)}^{t} p(s) d s \leq \frac{1}{e}$ and $\limsup _{t \rightarrow \infty} \int_{\tau(t)}^{t} p(s) d s<1$,

and for the second order equation, for instance when

$\liminf _{t \rightarrow \infty} \int_{\tau(t)}^{t} \tau(s) p(s) d s \leq \frac{1}{e}$ and $\lim _{t \rightarrow \infty} \int_{\tau(t)}^{t} \tau(s) p(s) d s<1$.

In another development, Li (1996) introduced a new technique to analyse the generalised characteristics equations to obtain some infinite integral conditions for $x^{\prime}(t)+p(t) x(t-\tau)=0$ oscillation of the nonautonomous delay differential equations. He considered the first order delay differential equation

where $p(t) \geq 0$ is a continuous function and $\tau$ is a positive constant, or the more general one

$x^{\prime}(t)+\sum_{i=1}^{n} p_{i}(t) x\left(t-\tau_{i}\right)=0$

where $p_{i}(t) \geq 0$ are continuous and $\tau_{i}$ are positive constants. Miyazaki (1996) studied the scalar equation $x^{\prime}(t)=p(t) x(t-\tau)$

where $\tau$ is a positive constant and $p:[0, \infty) \rightarrow \mathbf{R}$ is a continuous function. The following results were obtained by Miyazaki (1996);

(i) Suppose that $\tau<\omega / 4$. If $x(t)$ is not an oscillatory solution of equation (1.13), then

$\lim _{t \rightarrow \infty} x(t)=0$.

Also considering the case $p(t)=\sin t$, i.e.,

$x^{\prime}(t)=(\sin t) x(t-\tau)$

(ii) If $0<\tau \leq \pi / 6$, then every solution of (1.15)

of all solutions of the linear delay differential equation tends to zero as $t \rightarrow \infty$. Moreover, Ladas and Qian with positive and negative coefficients

(1990) obtained sufficient conditions for the oscillation

$$
y(t)+P(t) y(t-\tau)-Q(t) y(t-\sigma)=0
$$

where

$$
P, Q \in C\left[\left[t_{0}, \infty\right), \mathbf{R}^{+}\right] \text {and } \tau, \sigma \in[0, \infty) \text {. }
$$

Ladas and Qian (1990) further considered extensions of their work to include problems concerning neutral differential equations and some applications to the global asymptotic stability of the trivial solution. Elabbasy et al. (2000) presented infinite integral conditions for the oscillation of all solutions of first order delay differential equations with positive and

negative coefficients. Other studies include the work of Yuanji (1990) who studied the oscillation in the nonautonomous scalar differential equations with deviating arguments and got some oscillation criteria. As an application of the results, Yuanji (1990) proved a conjecture posed by Hunt and Yorke (1984), which was stated as follows; consider

$$
x^{\prime}(t)=-\sum_{i=1}^{n} q_{i}(t) x\left(t-T_{i}(t)\right)
$$

where $T_{i}$ and $q_{i}$ are continuous and positive valued on $[0, \infty)$.

if

$q_{i}(t) \geq \delta, T_{i}(t) \geq \delta$

holds and

$\liminf _{t \rightarrow \infty} \lambda^{-1} \sum_{1}^{n} q_{i}(t) \exp \left\{\lambda T_{i}(t)\right\}>1$,

then all solutions of equation (1.18) oscillate. Also Yuanji (1991) studied the oscillatory behaviour of first order differential equations with deviating arguments of the form

$x^{\prime}(t)+\sum_{1}^{n} p_{i} x\left(t-\tau_{i}\right)=0$ 
where $p_{i}, \tau_{i}, 1 \leq i \leq n$ are constants. In addition to that, he obtained necessary and sufficient conditions to guarantee that equation (1.21) is oscillatory, and also

$$
\lambda+\sum_{1}^{n} p_{i} e^{-\lambda \tau_{i}}=0
$$

discussed the equivalence of two kinds of oscillation for equation (1.21). He found that equation (1.21) is oscillatory if and only if the characteristic equation

has no real roots. Ladas and Stavroulakis (1982) studied the oscillatory behaviour of equations of the forms

$$
y^{\prime}(t)+\sum_{i=1}^{n} p_{i} y\left(t-\tau_{i}\right)=0
$$

and

$$
y^{\prime}(t)-\sum_{i=1}^{n} p_{i} y\left(t-\tau_{i}\right)=0,
$$

where $p_{i}$ and $\tau_{i}, i=1,2, \mathrm{~K}, n$ are positive constants. They proved that if each one of the conditions they established was satisfied then it implies that every solution of equations (1.23) and (1.24) oscillates. A generalization in the case where the

\section{Lemma 2.1 (Gyori and Ladas, 1991)}

Let $a \in(-\infty, 0), T \in(0, \infty), t_{0} \in R$ and suppose that a function $x(t) \in C\left[\left[t_{0}, \infty\right), R\right]$ satisfies the inequality

$$
x(t) \leq a+\max _{t-T \leq s \leq t} x(s) \text { for } t \geq t_{0} \text {, }
$$

then $x(t)$ cannot be a nonnegative function.

\section{Lemma 2.2 (Gyori and Ladas, 1991)}

Assume that

$q_{i}$ and $T_{i} \in C\left[\left[t_{0}, \infty\right), R^{+}\right]$for $i=1, \mathrm{~K}, n$.

Then the differential inequality

$$
x^{\prime}(t)+\sum_{i=1}^{n} q_{i}(t) x\left(t-T_{i}(t)\right) \leq 0, t \geq t_{0}
$$

has eventually positive solution if and only if the equation

$$
y^{\prime}(t)+\sum_{i=1}^{n} q_{i}(t) y\left(t-T_{i}(t)\right)=0, t \geq t_{0}
$$

has an eventually positive solution.

A similar result holds for advanced delay differential inequalities. Thus

Lemma 2.3 (Gyori and Ladas, 1991)

Assume that equation (2.2) holds and suppose that the differential inequality

$$
x^{\prime}(t)-\sum_{i=1}^{n} q_{i}(t) x\left(t+T_{i}(t)\right) \geq 0
$$

has an eventually positive solution. Then the equation

$$
y^{\prime}(t)-\sum_{i=1}^{n} q_{i}(t) y\left(t+T_{i}(t)\right)=0
$$

also has an eventually positive solution. Proofs of lemmas (2.1), (2.2) and (2.3) are found in Gyori and Ladas (1991).

\section{Lemma 2.4 ( $\mathrm{Li}, 1996)$}

Consider the delay differential equation

$$
x^{\prime}(t)+\sum_{i=1}^{n} R_{i}(t) x\left(t-\tau_{i}\right)=0, t \geq t_{0}
$$

and assume that $\limsup _{t \rightarrow \infty} \int_{t}^{t+\tau_{i}} R_{i}(s) d s>0$ for some $i$ and $x(t)$ is an eventually positive solution of equation (2.7), then for the same $i$, 
$\liminf _{t \rightarrow \infty} \frac{x\left(t-\tau_{i}\right)}{x(t)}<\infty$.

Lemma 2.5 ( $(\mathrm{Li}, 1996)$

If equation (2.7) has an eventually positive solution, then

$\int_{t}^{t+\tau_{i}} R_{i}(s) d s<1, i=1, \mathrm{~K}, n$

eventually. Li (1996) gave the proofs of lemmas (2.4) and (2.5).

Also consider the first order delay differential equation

$x^{\prime}(t)+P(t) x(t-\sigma)-Q(t) x(t-\tau)=0$,

where $P(t)$ and $Q(t)$ are positive continuous real functions and $\sigma, \tau$ are positive constants. The following statement holds;

Lemma 2.6 (Elabbasy et al., 2000)

Assume that for

$R(t)=P(t)-Q(t+\tau-\sigma)$,

(i) $\int_{t}^{t+\sigma} R(s) d s>0, t \geq t_{0}$ for some $t_{0}>0$.

(ii) $\int_{t_{0}}^{\infty} R(t) \ln \left[e \int_{t}^{t+\sigma} R(s) d s\right] d t=\infty$.

Then every solution of equation (2.10) oscillates.

Consider the generalized first order delay differential equation

$x^{\prime}(t)+\sum_{i=1}^{n} P_{i}(t) x\left(t-\sigma_{i}\right)-\sum_{j=1}^{m} Q_{j}(t) x\left(t-\tau_{j}\right)=0$,

where $P_{i}(t), Q_{j}(t) \in C\left(\left[t_{0}, \infty\right), R^{+}\right) \quad$ and $\quad \sigma_{i}, \tau_{j} \in[0, \infty) \quad, \quad$ for $\quad i=1, \mathrm{~K}, n \quad$ and $j=1, \mathrm{~K}, m$. The following statement holds;

Lemma 2.7 (Elabbasy et al., 2000)

Assume that all conditions from lemma (2.6) are satisfied, $\sigma_{p}=\max \left\{\sigma_{1}, \sigma_{2}, \sigma_{3}, \mathrm{~K}, \sigma_{p}\right\}, \sum_{i=1}^{p} \int_{t}^{t+\sigma_{i}} R_{i}(s) d s>0$ for $t \geq t_{0}$ for some $t_{0}>0$. Also assume that

(iii) $\limsup _{t \rightarrow \infty} \int_{t}^{t+\sigma_{p}} R_{p}(s) d s>0$.

(iv) $\int_{t_{0}}^{\infty}\left(\sum_{i=1}^{p} R_{i}(t)\right) \ln \left[e \sum_{i=1}^{p} \int_{t}^{t+\sigma_{i}} R_{s}(s) d s\right] d t=\infty$

where $R_{i}(t)=P_{i}(t)-\sum_{k \in J_{i}} Q_{k}\left(t+\tau_{k}-\sigma_{i}\right)$. Then every solution of equation (2.12) oscillates. Lemma (2.6) and lemma (2.7) were proved in Elabbasy et al. (2000).

\section{RESULTS}

The aim of this paper is to present a more general form of delay differential equations that involve both positive and negative coefficients and obtain as main results an entirely new set of infinitely integral Consider the delay differential equations

$x^{\prime}(t)+\sum_{i=1}^{1} q_{i}(t) x\left(t-T_{i}(t)\right)-\sum_{j=1}^{r} q_{j}(t) x\left(t-T_{j}(t)\right)=0$

where $q_{i}(t), q_{j}(t), T_{i}(t)$ and $T_{j}(t)$ are continuous and positive valued on $[0, \infty)$.

It is important to note that the two new theorems presented here give a detailed description of the oscillatory solution of equation (3.1) for infinite integral conditions. Theorem (3.1) explains that if certain conditions for every solution of oscillation of equation (3.1) below. We categorise the results obtain in this section in form of theorems (3.1) and (3.2) respectively. These established theorems are what we have achieved and considered as our results. conditions are satisfied then $x(t)$ cannot be a nonnegative function, while Theorem (3.2) describes in detail that every solution of equation (3.1) oscillates. Thus; 
BAJOPAS Volume 11 Number 2 December, 2018

Theorem 3.1. Consider equation (3.1). Assume that all conditions from lemma (2.1) are satisfied and suppose that the inequality

$x(t) \leq c+\left[\max _{t-T_{i} \leq s \leq t}(s)+\max _{t-T_{j} \leq s \leq t}(s)\right], t \geq t_{0}$

then $x$ cannot be a nonnegative function.

Proof: For the sake of contradiction, let assume that $x(t) \geq 0$ for $t \geq t_{0}$. First we claim that $x(t)$ is a bounded function. Otherwise there exists a $t_{1} \geq t_{0}$ such that

$x\left(t_{1}\right)=\left[\max _{t_{0}-T_{i} \leq s \leq t_{1}}(s)+\max _{t_{0}-T_{j} \leq s \leq t_{1}}(s)\right]$.

Then equation (3.2) yields

$x\left(t_{1}\right) \leq c+\left[\max _{t_{1}-T_{i} \leq s \leq t_{1}}(s)+\max _{t_{1}-T_{j} \leq s \leq t_{1}}(s)\right] \leq c+x\left(t_{1}\right)<x\left(t_{1}\right)$,

which is a contradiction. Hence setting

$M=\lim _{t \rightarrow \infty} \sup x(t)<\infty$.

It now follows from equation (3.2) that

$M \leq c+M<M$,

which is not possible. Hence the proof is complete.

Consider equation (3.1) and the general advanced delay differential equation with mixed argument

$y^{\prime}(t)+\sum_{i=1}^{1} q_{i}(t) y\left(t-T_{i}(t)\right)+\sum_{j=1}^{r} q_{j}(t) y\left(t+T_{j}(t)\right)=0, t \geq t_{0}$

where $q_{i}(t), q_{j}(t) \in C\left(\left[t_{0}, \infty\right), R^{+}\right) \quad$ and $\quad T_{i}, T_{j} \in[0, \infty) \quad$ for $\quad i=1,2, \mathrm{~K}, 1 \quad$ and $j=1,2, \mathrm{~K}, r$. By solution of equation (3.7) we mean a function $y(t) \in C\left(\left[t_{0}-\rho\right), R\right)$ that for some $t_{0}$ satisfies equation (3.7) for all $t \geq t_{0}$, where $\rho=\max \left\{\max _{1 \leq i \leq 1} T_{i}, \max _{1 \leq j \leq r} T_{j}\right\}$.

Considering equation (3.7) above, the result below indicates that every solution of equation (3.1) oscillates. this completes the proof.

Theorem 3.2. Assume that all conditions from lemmas (2.1), (2.2) and (2.3) are satisfied. Let

$\lambda(t)=\frac{-y^{\prime}(t)}{y(t)}$

be nonnegative and continuous, and also there exists $t_{1} \geq t_{0}$ such that $y\left(t_{1}\right)>0$ and $y(t)=y\left(t_{1}\right) e^{\int_{t_{1}}^{t} \lambda(s) d s-\int_{t_{1}}^{t} \lambda(\sigma) d \sigma}$. If the advanced delay differential inequality

$z^{\prime}(t)+\sum_{i=1}^{1} q_{i}(t) z\left(t-T_{i}(t)\right)+\sum_{j=1}^{r} q_{j}(t) z\left(t+T_{j}(t)\right) \geq 0, t \geq t_{0}$

has an eventually positive solution, then every solution of equation (3.1) oscillates.

Proof: Theorem (3.1) indicates that the solution $x(t)$ to equation (3.1) cannot be a nonnegative function. Assume the contrary that equation (3.1) has an eventually positive solution $x(t)$. Then by lemma (2.3) we have that the inequality (3.9) has an eventually positive solution if and only if equation (3.7) also has an eventually positive solution.

If equation (3.8) holds and further assume that $\lambda(t)$ satisfies the generalized characteristic equation

$\lambda(t)=\sum_{i=1}^{1} q_{i}(t) e^{\int_{t-T_{i}}^{t} \lambda(s) d s}-\sum_{j=1}^{r} q_{j}(t) e^{-\int_{t}^{t+T_{j}} \lambda(\sigma) d \sigma}$,

$i=1,2, \mathrm{~K}, 1$ and $j=1,2, \mathrm{~K}, r$. 


\section{BAJOPAS Volume 11 Number 2 December, 2018}

By lemma (2.6) and the fact that

$$
e^{r x} \geq x+\frac{\ln (e r)}{r} \text { for } r>0 \text {, }
$$

we can have $\lambda(t)$ to be

$$
\begin{aligned}
& \lambda(t)=\sum_{i=1}^{1} q_{i}(t) e^{\left(\sum_{i=1}^{1} \int_{t-T_{i}}^{t} q_{i}(s) d s \cdot \frac{1}{\sum_{i=1}^{1} \int_{t-T_{i}}^{t} q_{i}(s) d s} \cdot \int_{t-T_{i}}^{t} \lambda(s) d s\right.}-\sum_{j=1}^{r} q_{j}(t) e^{-\left(\sum_{j=1}^{r} \int_{t}^{t+T_{j}} q_{j}(\sigma) d \sigma \cdot \frac{1}{\sum_{j=1}^{r} \int_{t}^{t+T_{j}} q_{j}(\sigma) d \sigma} \cdot \int_{t}^{t+T_{j}} \lambda(\sigma) d \sigma\right)} \\
& \geq \sum_{i=1}^{1} q_{i}(t)\left[\frac{1}{\sum_{i=1}^{1} \int_{t-T_{i}}^{t} q_{i}(s) d s} \cdot \int_{t-T_{i}}^{t} \lambda(s) d s+\frac{\ln \left(e \sum_{i=1}^{1} \int_{t-T_{i}}^{t} q_{i}(s) d s\right)}{\sum_{i=1}^{1} \int_{t-T_{i}}^{t} q_{i}(s) d s}\right] \\
& +\sum_{j=1}^{r} q_{j}(t)\left[\frac{1}{\sum_{j=1}^{r} \int_{t}^{t+T_{j}} q_{j}(\sigma) d \sigma} \cdot \int_{t}^{t+T_{j}} \lambda(\sigma) d \sigma-\frac{\ln \left(e \sum_{j=1}^{r} \int_{t}^{t+T_{j}} q_{j}(\sigma) d \sigma\right)}{\sum_{j=1}^{r} \int_{t}^{t+T_{j}} q_{j}(\sigma) d \sigma}\right]
\end{aligned}
$$

This implies that

$$
\begin{aligned}
& \lambda(t) \cdot \sum_{i=1}^{1} \int_{t-T_{i}}^{t} q_{i}(s) d s \cdot \sum_{j=1}^{r} \int_{t}^{t+T_{j}} q_{j}(\sigma) d \sigma-\sum_{i=1}^{1} q_{i}(t) \cdot \sum_{j=1}^{r} \int_{t}^{t+T_{j}} q_{j}(\sigma) d \sigma \cdot \int_{t-T_{i}}^{t} \lambda(s) d s \\
& -\sum_{j=1}^{r} q_{j}(t) \cdot \sum_{i=1}^{1} \int_{t-T_{i}}^{t} q_{i}(s) d s \cdot \int_{t}^{t+T_{j}} \lambda(\sigma) d \sigma \geq \sum_{i=1}^{1} q_{i}(t) \cdot \ln \left(e \sum_{i=1}^{1} \int_{t-T_{i}}^{t} q_{i}(s) d s\right) \\
& \cdot \sum_{j=1}^{r} \int_{t}^{t+T_{j}} q_{j}(\sigma) d \sigma-\sum_{j=1}^{r} q_{j}(t) \cdot \ln \left(e \sum_{j=1}^{r} \int_{t}^{t+T_{j}} q_{j}(\sigma) d \sigma\right) \cdot \sum_{i=1}^{1} \int_{t-T_{i}}^{t} q_{i}(s) d s(3.13)
\end{aligned}
$$

Taking the integral of both sides of equation (3.13) from $T$ to $N$, for $N>T$, we have

$$
\begin{aligned}
& \int_{T}^{N} \lambda(t)\left(\sum_{i=1}^{1} \int_{t-T_{i}}^{t} q_{i}(s) \cdot \sum_{j=1}^{r} \int_{t}^{t+T_{j}} q_{j}(\sigma) d \sigma d s\right) d t \\
& -\int_{T}^{N} \sum_{i=1}^{1} q_{i}(t)\left(\sum_{j=1}^{r} \int_{t}^{t+T_{j}} q_{j}(\sigma) \cdot \int_{t-T_{i}}^{t} \lambda(s) d s d \sigma\right) d t \\
& -\int_{T}^{N} \sum_{j=1}^{r} q_{j}(t)\left(\sum_{i=1}^{1} \int_{t-T_{i}}^{t} q_{i}(s) \cdot \int_{t}^{t+T_{j}} \lambda(\sigma) d \sigma d s\right) d t \\
& \geq \int_{T}^{N} \sum_{i=1}^{1} q_{i}(t)\left(\ln \left(e \sum_{i=1}^{1} \int_{t-T_{i}}^{t} q_{i}(s) d s\right) \cdot \sum_{j=1}^{r} \int_{t}^{t+T_{j}} q_{j}(\sigma) d \sigma\right) d t \\
& -\int_{T}^{N} \sum_{j=1}^{r} q_{j}(t)\left(\ln \left(e \sum_{j=1}^{r} \int_{t}^{t+T_{j}} q_{j}(\sigma) d \sigma\right) \cdot \sum_{i=1}^{1} \int_{t-T_{i}}^{t} q_{i}(s) d s\right) d t .
\end{aligned}
$$

Interchanging the order of integration, and expressing the terms on the left hand side of inequality (3.14) only, we find that

$$
\int_{T}^{N} \sum_{i=1}^{1} q_{i}(t)\left(\sum_{j=1}^{r} \int_{t}^{t+T_{j}} \int_{t-T_{i}}^{t} q_{j}(\sigma) \cdot \lambda(s) d s d \sigma\right) d t
$$




$$
\begin{aligned}
& \geq \int_{T}^{N-T_{i}}\left(\int_{s}^{s+T_{i}} \int_{s-T_{j}}^{s} \sum_{i=1}^{1} q_{i}(t) \cdot \sum_{j=1}^{r} q_{j}(\sigma) \cdot \lambda(s) d \sigma d t\right) d s \\
& +\int_{T}^{N-T_{j}}\left(\int_{s}^{s+T_{j}} \int_{s-T_{i}}^{s} \sum_{j=1}^{r} q_{j}(t) \cdot \sum_{i=1}^{1} q_{i}(s) \cdot \lambda(\sigma) d t d s\right) d \sigma .
\end{aligned}
$$

Hence

$$
\begin{aligned}
& \int_{T}^{N}\left(\sum_{i=1}^{1} q_{i}(t)\right) \cdot \sum_{j=1}^{r} \int_{t}^{t+T_{j}} \int_{t-T_{i}}^{t} q_{j}(\sigma) \cdot \lambda(s) d s d \sigma d t \\
& \geq \int_{T}^{N-T_{i}} \lambda(s)\left(\int_{s}^{s+T_{i}} \int_{s-T_{j}}^{s} \sum_{i=1}^{1} q_{i}(t) \cdot \sum_{j=1}^{r} q_{j}(\sigma) d \sigma d t\right) d s \\
& +\int_{T}^{N-T_{j}} \lambda(\sigma)\left(\int_{s}^{s+T_{j}} \int_{s-T_{i}}^{s} \sum_{j=1}^{r} q_{j}(t) \cdot \sum_{i=1}^{1} q_{i}(s) d t d s\right) d \sigma .
\end{aligned}
$$

Then

$$
\begin{aligned}
& \int_{T}^{N}\left(\sum_{i=1}^{1} q_{i}(t)\right) \cdot \sum_{j=1}^{r} \int_{t}^{t+T_{j}} \int_{s-T_{i}}^{t} q_{j}(\sigma) \lambda(s) d s d \sigma d t \\
& \geq \sum_{i=1}^{1} \int_{T}^{N-T_{i}} \lambda(t)\left(\int_{t}^{t+T_{i}} \int_{s-T_{j}}^{s} q_{i}(s) \cdot \sum_{j=1}^{r} q_{j}(\sigma) d \sigma d s\right) d t \\
& +\sum_{j=1}^{r} \int_{T}^{N-T_{j}} \lambda(t)\left(\int_{t}^{t+T_{j}} \int_{s-T_{i}}^{s} q_{j}(\sigma) \cdot \sum_{i=1}^{1} q_{i}(s) d s d \sigma\right) d t .
\end{aligned}
$$

It follows from equation (3.14) and equation (3.17) that

$$
\begin{aligned}
& \int_{T}^{N} \lambda(t)\left(\sum_{i=1}^{1} \int_{t-T_{i}}^{t} \sum_{j=1}^{r} \int_{t}^{t+T_{j}} q_{i}(s) \cdot q_{j}(\sigma) d \sigma d s\right) d t \\
& -\left[\int_{T}^{N-T_{i}} \lambda(t)\left(\int_{t}^{t+T_{i}} \int_{s-T_{j}}^{s} \sum_{i=1}^{1} q_{i}(s) \cdot \sum_{j=1}^{r} q_{j}(\sigma) d \sigma d s\right) d t\right. \\
& \left.+\int_{T}^{N-T_{j}} \lambda(t)\left(\int_{t}^{t+T_{j}} \int_{s-T_{i}}^{s} \sum_{j=1}^{r} q_{j}(\sigma) \cdot \sum_{i=1}^{1} q_{i}(s) d s d \sigma\right) d t\right] \\
& \geq \int_{T}^{N} \sum_{i=1}^{1} q_{i}(t)\left(\ln e \sum_{i=1}^{1} \int_{t-T_{i}}^{t} q_{i}(s) \cdot \sum_{j=1}^{r} \int_{t}^{t+T_{j}} q_{j}(\sigma) d \sigma d s\right) d t \\
& -\int_{T}^{N} \sum_{j=1}^{r} q_{j}(t)\left(\ln e \sum_{j=1}^{r} \int_{t}^{t+T_{j}} q_{j}(\sigma) \cdot \sum_{i=1}^{1} \int_{t-T_{i}}^{t} q_{i}(s) d s d \sigma\right) d t .
\end{aligned}
$$

$$
\begin{aligned}
& \text { Hence } \\
& \sum_{i=1}^{1} \int_{N-T_{i}}^{N} \lambda(t)\left(\int_{t}^{t+T_{i}} \int_{s-T_{j}}^{s} q_{i}(s) \cdot \sum_{j=1}^{r} q_{j}(\sigma) d \sigma d s\right) d t \\
& +\sum_{j=1}^{r} \int_{N-T_{j}}^{N} \lambda(t)\left(\int_{t}^{t+T_{j}} \int_{s-T_{i}}^{s} q_{j}(\sigma) \cdot \sum_{i=1}^{1} q_{i}(s) d s d \sigma\right) d t \\
& \geq \int_{T}^{N}\left(\sum_{i=1}^{1} q_{i}(t)\right)\left(\ln e \sum_{i=1}^{1} \int_{t-T_{i}}^{t} q_{i}(s) \cdot \sum_{j=1}^{r} \int_{t}^{t+T_{j}} q_{j}(\sigma) d \sigma d s\right) d t \\
& -\int_{T}^{N}\left(\sum_{j=1}^{r} q_{j}(t)\right)\left(\ln e \sum_{j=1}^{r} \int_{t}^{t+T_{j}} q_{j}(\sigma) \cdot \sum_{i=1}^{1} \int_{t-T_{i}}^{t} q_{i}(s) d s d \sigma\right) d t .
\end{aligned}
$$




$$
\int_{t}^{t+T_{i}} \int_{s-T_{j}}^{s} q_{i}(s) \cdot \sum_{j=1}^{r} q_{j}(\sigma) d \sigma d s<1, i=1,2, \mathrm{~K}, 1
$$

and

$$
\int_{t}^{t+T_{j}} \int_{s-T_{i}}^{s} q_{j}(\sigma) \cdot \sum_{i=1}^{1} q_{i}(s) d s d \sigma<1, j=1,2, \mathrm{~K}, r
$$

eventually.

Then by equations (3.19), (3.20), and (3.21) we find that

$$
\begin{aligned}
& {\left[\sum_{i=1}^{1} \int_{N-T_{i}}^{N} \lambda(t)+\sum_{j=1}^{r} \int_{N-T_{j}}^{N} \lambda(t)\right] d t} \\
& \geq \int_{T}^{N}\left(\sum_{i=1}^{1} q_{i}(t)\right) \ln \left(e \sum_{i=1}^{1} \int_{t-T_{i}}^{t} q_{i}(s) \cdot \sum_{j=1}^{r} \int_{t}^{t+T_{j}} q_{j}(\sigma) d \sigma d s\right) d t \\
& -\int_{T}^{N}\left(\sum_{j=1}^{r} q_{j}(t)\right) \ln \left(e \sum_{j=1}^{r} \int_{t}^{t+T_{j}} q_{j}(\sigma) \cdot \sum_{i=1}^{1} \int_{t-T_{i}}^{t} q_{i}(s) d s d \sigma\right) d t .
\end{aligned}
$$

Or

$$
\begin{aligned}
& \sum_{i=1}^{1} \ln \frac{y\left(N-T_{i}\right)}{y(N)}+\sum_{j=1}^{r} \ln \frac{y\left(N-T_{j}\right)}{y(N)} \\
& \geq \int_{T}^{N}\left(\sum_{i=1}^{1} q_{i}(t)\right) \ln \left(e \sum_{i=1}^{1} \int_{t-T_{i}}^{t} q_{i}(s) \cdot \sum_{j=1}^{r} \int_{t}^{t+T_{j}} q_{j}(\sigma) d \sigma d s\right) d t \\
& -\int_{T}^{N}\left(\sum_{j=1}^{r} q_{j}(t)\right) \ln \left(e \sum_{j=1}^{r} \int_{t}^{t+T_{j}} q_{j}(\sigma) \cdot \sum_{i=1}^{1} \int_{t-T_{i}}^{t} q_{i}(s) d s d \sigma\right) d t .
\end{aligned}
$$

However, in view of (iv) from lemma (2.7), we have

$$
\lim _{t \rightarrow \infty} \prod_{i=1}^{1} \frac{y\left(t-T_{i}\right)}{y(t)}+\lim _{t \rightarrow \infty} \prod_{j=1}^{r} \frac{y\left(t-T_{j}\right)}{y(t)}=\infty .
$$

Therefore, by implication, this means that

$\lim _{t \rightarrow \infty} \frac{y\left(t-T_{1}\right)}{y(t)}+\lim _{t \rightarrow \infty} \frac{y\left(t-T_{r}\right)}{y(t)}=\infty$.

Moreover, by lemma (2.4) we have

$\liminf _{t \rightarrow \infty} \frac{y\left(t-T_{1}\right)}{y(t)}+\liminf _{t \rightarrow \infty} \frac{y\left(t-T_{r}\right)}{y(t)}<\infty$.

This contradicts equation (3.25) and the proof is complete. Therefore, every solution of equation (3.1) oscillates. That ends the proof.

\section{DISCUSSION}

As earlier mentioned, this work is motivated by the work of $\mathrm{Li}$ (1996) where integral conditions for oscillation of nonautonomous delay differential equations were obtained. We observed that most of the conditions from Li (1996) and other similar works concentrated on positive coefficients of the delay equations as highlighted in section (1) above. Although some considered nonautonomous delay differential equations with both positive and negative coefficients like Ladas and Qian (1990) and Elabbasy et al. (2000), theorems (3.1) and (3.2) we obtained are considered as improvements on their respective conditions for oscillation of equation (3.1) where $p_{i}, i=1,2, \mathrm{~K}, n$ and $q_{j}, j=1,2, \mathrm{~K}, 1$ are continuous on specified intervals.
We also discovered that based on the newly established results, that certain restrictions associated with some of the existing gaps as we identified in the literature are addressed with introduction of the new set of infinite integral conditions for oscillation of delay differential equations with positive and negative coefficients. For instance, if $q=0$ in equation (3.1), then the equation is similar to any of the equations (1.12), (1.21) and (1.23) by Li (1996), Yuanji (1991) and Stavroulakis (1982) respectively. Consequently, all conditions for the solutions of equations (1.12), (1.21) and (1.23) are satisfied respectively by our new set of conditions. Attempts to impose any of the conditions by Li (1996), Yuanji (1991) and Stavroulakis (1982) respectively to equation (3.1) will fail to be satisfied. 


\section{CONCLUSION}

The key objectives of this paper are being addressed in section (3.0). This include formulations and proves of theorems (3.1) and (3.2), which gave detailed description of the oscillatory solution of equation (3.1)

\section{REFERENCES}

Barr, T. H. (1995) Oscillations in Linear Delay Differential Equations. Journal of Mathematical Analysis and Applications, 19, 261-277.

Elabbasy, E. M., Hegazi, A. S., Saker, S. H. (2000) Oscillation of Solutions to Delay Differential Equations with Positive and Negative Coefficients. Electronic Journal of Differential Equations, 2000 (2000), 1-13.

Gyori, I., Ladas, G. (1991) Oscillation Theory of Delay Differential Equations with Applications. The Clarendon Press, Oxford University Press, New York.

Hunt, B. R., Yorke, J. A. (1984) When All Solutions of $x^{\prime}=-\sum q_{i}(t) x\left(t-T_{i}(t)\right)$

Oscillate. Journal of Differential Equations, 53, 139-145.

Ladas, G., Qian, C. (1990) Oscillation in Differential Equations with Positive and Negative Coefficients. Canadian Mathematical Bulletin, 33, 442-451.

Ladas, G., Stavroulakis, I. P. (1982) Oscillations Caused by Several Retarded and Advanced Arguments. Journal of Differential Equations, 44, 134-152. for infinite integral conditions. The new set of conditions established for first order linear delay differential equations with positive and negative coefficients are similar to those indicated in the literature with more generalized forms.

Li, B. (1996) Oscillation of First Order Delay Differential Equations. In: Proceedings of the American Mathematical Society, pp. 3729-3737.

Miyazaki, R. (1996) Asymptotic Behaviour for Linear Delay Differential Equations with Periodically Oscillatory Coefficients. Journal of Mathematical Analysis and Applications, 204, 183-205.

Philos, C. G., Sficas, Y. G. (1998) An Oscillation Criterion for First Order Linear Delay Differential Equations. Canadian Mathematical Bulletin, 41, 207-213.

Stavroulakis, I. P. (2004) Oscillation Criteria for Functional Differential Equations. In: Conference on Differential Equations and Application in Mathematical Biology, Electronic Journal of Differential Equations, Conference, 12, pp. 171-180.

Yuanji, C. (1990) Oscillation in Nonautonomous Scalar Differential Equations with Deviating Arguments. In: Proceedings of the American Mathematical Society, pp. 711-719.

Yuanji, C. (1991) Oscillation Criteria for First Order Differential Equations with Deviating Arguments. Journal of Mathematical Analysis and Applications, 155, 572-588. 